\title{
Sampling schemes for estimating root density distribution in cropped fields
}

\author{
Meine van Noordwijk, Johan Floris and Anton de Jager \\ Institute for Soil Fertility, P.O. Box 30003, 9750 RA Haren (Gr), Netherlands \\ Received 4 December 1984; accepted 14 March 1985
}

Key-words: root research methods, pinboard, auger, profile wall counts, shoot:root ratio, bias, grassland, cereals, sugar-beet, potato

\section{Summary}

In discussions on root research methods, so far the choice of sampling schemes seems to have been a neglected topic. In this paper sampling schemes are discussed for various situations. It is shown that the schemes used traditionally for row crops such as cereals may give a bias of about $30 \%$ in total root dry weight. More reliable sampling schemes are presented, as well as possibilities for correction of data from traditional sampling.

Available data on variation in root mass per auger sample are summarized to calculate the number of replicate samples required to detect differences (of given magnitude) between two means. The coefficient of variation of root weight per auger sample seems to be fairly constant at around $40 \%$ in grassland, with slightly higher values for deeper layers of soil.

A method is described by which slices of a root system, obtained by the pinboard method, can be used to estimate the total size of the root system of a single plant. As an example results are discussed for a potato experiment.

A related technique is presented for calculations on profile wall observations.

\section{Introduction}

Root systems of plants can be described in two systems of reference, viz the soil or the plant, with root length, surface area, volume or weight as the root parameter depending on specific research questions. Root length per unit volume of soil can for example be used for theoretical estimates of the part of the potentially available resources (water and nutrients) which can actually be taken up by the root system at the required rate (van Noordwijk, 1983); input of root dry matter per unit volume of soil is important in the description of soil life. The total size of the root system per plant is important, as this integrates all possibilities for uptake of a root system, or reflects dry-matter distribution over shoots or roots (Brouwer, 1984). In tube or container experiments the complete root system of a single plant can be washed out 
(Schuurman \& Goedewaagen, 1971); in the field this is rarely possible, as the roots of a plant usually are dispersed over a large volume of soil and are intermingled with the roots of neighbouring plants.

For crop plants with a regular plant spacing, the average total amount of roots per plant can be calculated if the root distribution per unit volume of soil is known. In the case of regular plant spacing in monocultures, the cropped surface area per plant is a rectangle, which we call 'unit soil area' (Fig. 1A). The unit soil area can be expected to contain just as many roots of neighbouring plants as there are roots of the plant studied outside the unit soil area (Fig. 1B). The unit soil area consists of four parts of equal size, divided by two planes of symmetry, which may differ, however, in degree of shadedness of the soil. Knowledge of the root density distribution in this 'unit soil area' can be used for both ways of describing root systems.

For row crops with dense spacing in the row a 'unit soil strip' can be defined, which consists of the unit soil areas of all plants in the same row. The 'unit soil strip' is bounded by the lines midway between neighbouring rows, and consists of two symmetrical parts.

Sampling methods which are commonly used to describe root density distribution are (see Böhm, 1979, for details):

(a) cylindrical auger samples from which roots are washed free;

(b) cylindrical auger samples that are broken by hand to count the number of roots on the two breakage faces;

(c) large rectangular soil monoliths, usually on pinboards to wash large 'slices' of root systems;

(d) smaller blocks of soil, either obtained directly in the field or by subdividing larger monoliths;

(e) root counts on vertical (or horizontal), smoothed surfaces in the field (profiles),

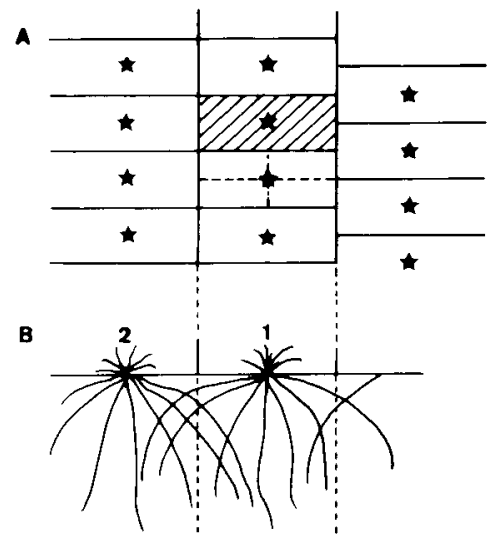

Fig. 1. Unit soil area for regular plant spacing. A: a top view, asterisks denoting plants, lines denoting planes of symmetry, and the shaded area the 'unit soil area' which consists of four equal parts. B: a side view of the roots in the soil profile, showing an equal number of roots of plant 1 and 2 crossing the boundary between their respective unit soil areas. 
either directly or after mapping the roots on plastic sheets.

The unit soil area is rectangular (for regular plant spacing), so rectangular samples (d) are best to represent this block of soil. For practical reasons, however, cylindrical samples ( $a$ and $b$ ) are used most frequently, as they can be replicated throughout the field without disturbing the sampling sites more than absolutely necessary. In the following chapter we will describe, for auger samples, the effect of various sampling schemes on the calculated average root density per unit volume. The problem is how to describe the essentially rectangular unit soil area with round samples.

For irregular plant spacings (mixed cultures and natural vegetation) the 'unit soil area' approach cannot be used as such. Statements about the size of the root system of an average plant will be meaningless in such a case. Other techniques are necessary to determine the total size of the root system of a single plant and to describe the variation in root density per unit volume of soil.

An alternative method to estimate the total size of the root system of a plant is to use large 'slices' of the root system on a pinboard and to remove roots of neighbouring plants after washing. Assuming radial symmetry, root density per unit volume of soil can be measured for all positions present on the pinboard and can be extrapolated to the corresponding ring around the plant, by multiplication with the relevant soil volume. In fact, rectangular samples are now used to estimate root densities in an assumed circular pattern, for which circle-sector samples would be a better choice. In the chapter dealing with pinboard techniques we will discuss problems and possibilities of this approach. Related to this technique, an alternative procedure is presented to estimate root length density from profile wall observations (e).

The difficulties met in choosing well-designed sampling schemes depend on the heterogeneity of the root distribution. Such heterogeneity may arise from various causes, the soil, the plant or their interaction. Local variation in rootability of the soil (e.g. in aggregated soils), the branching pattern of roots causing local clusters of branch roots (which may be especially important in deeper layers of the profile) and the stimulation of branch root development near localized nutrient supplies are examples of these three types of heterogeneity. A distinction between these situations can only be made by combining root data with soil data obtained at the same sample scale. The branching pattern of the roots is a plant characteristic of obvious importance. Fig. 2 gives a schematic view of the distribution of the main roots of some agriculturally important plants. The spatial distribution of their root systems becomes more complex in the order: grasses, cereals, sugar-beet, potato. In grassland, root density varies essentially with depth only. For row crops with a dense spacing in the row, such as cereals, the pattern can be essentially two-dimensional. Wider plant spacing in the row causes the pattern to be three-dimensional. Special problems are encountered in a crop like sugar-beet, because the branch roots are concentrated on two sides of the taproot (like a beard). In potato fields ridges and troughs give rise to additional heterogeneity.

Considerable variation among samples is well known in root research. Often this variation is only seen as troublesome, as large numbers of replicate samples are required to obtain reasonably accurate estimates of the average root density. The 


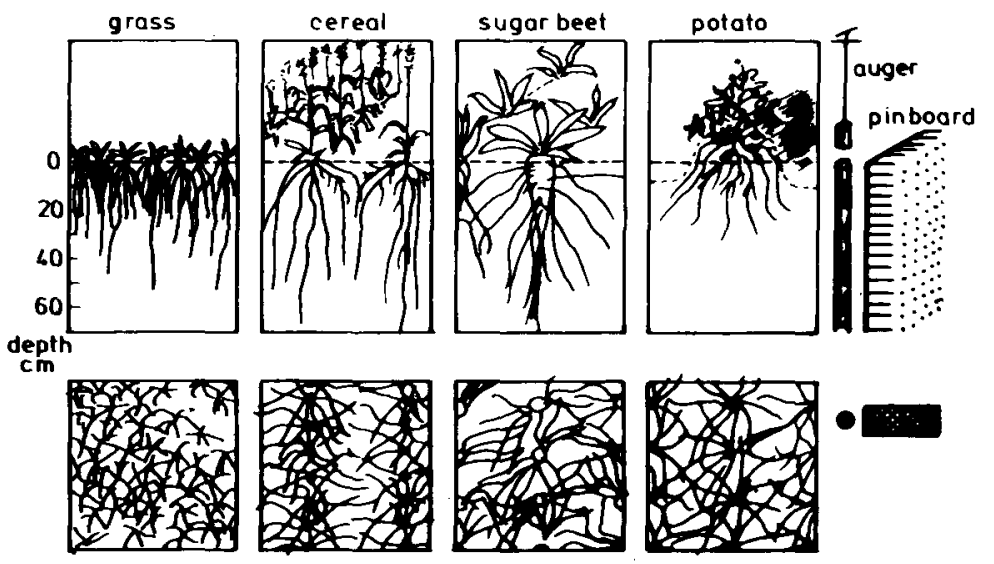

Fig. 2. Schematic representation of root pattern of four major agricultural crops, as seen in a profile wall and as seen from above.

variation in local root density, which is reflected in sample variation, is an important aspect in itself. As the relationship between local root density and possibilities for uptake usually is not linear but rather shaped like a saturation curve (van Noordwijk, 1983) the average root density does not provide sufficient information if the statistical distribution of sample values is unknown. Especially the frequency of low values is important, as these are related to parts of the soil resources relatively out of reach of the root system. In this context, describing the pattern in local variation of root density is a relevant task in itself. The recent trend in agriculture of deteriorating soil structure due to the use of heavy machinery probably increases soil heterogeneity and therefore heterogeneity in root distribution.

In describing variation in local root density, sample size is an important aspect. Depending on the mobility of nutrients and water, root heterogeneity and pattern can be relevant on various scales (de Willigen \& van Noordwijk, in prep.). Root maps have the advantage that patterns can be measured at any scale, while in all other methods the choice of the (sub-)sample size determines the scale on which heterogeneity and pattern will be observed.

\section{Sampling with auger techniques}

\section{Grassland}

When sampling in established grassland, the sampling sites can be chosen randomly with regard to the distance to the nearest plant. The number of samples which has to be taken depends on the required precision in estimating average root density and on the variation between samples. Assuming a normal distribution of root sample data (and preliminary tests have shown this assumption to be acceptable) and average root densities for two situations can be subjected to a t-test, provided the variance for the two data sets is similar. For such cases Eq. 1 gives the relationship between the required number of replicates $n$ (for each of the two objects), the coeffi- 
number of replicates

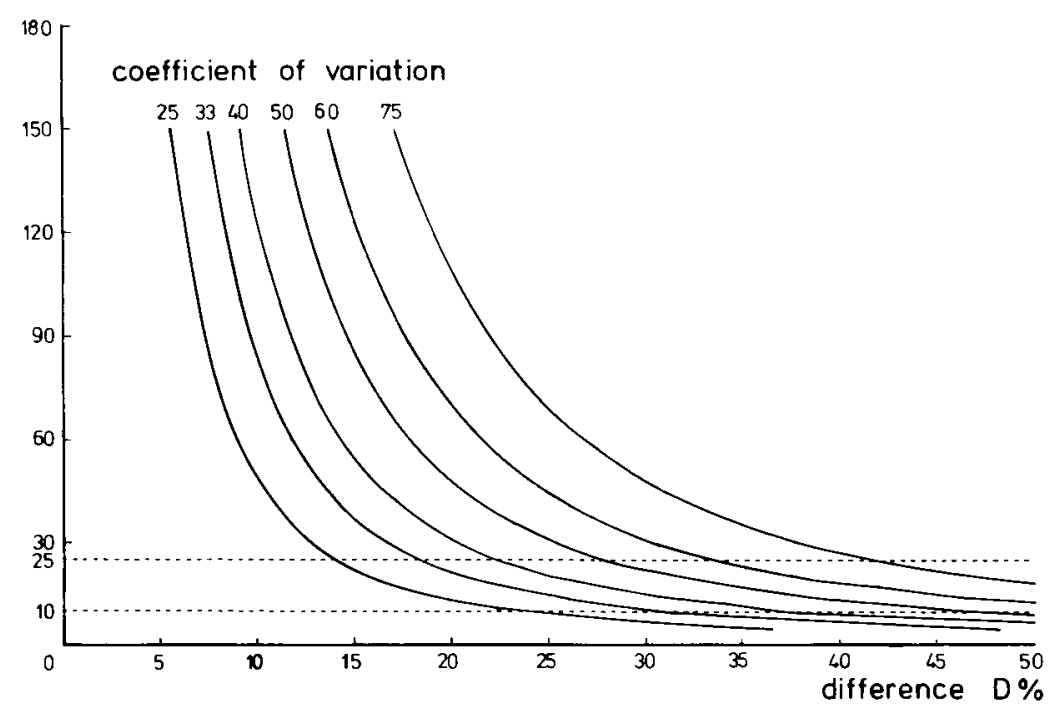

Fig. 3. Minimum number of replicates required to distinguish between two means in a two-sided t-test at $95 \%$ significance level, according to Equation 1.

cient of variation (standard deviation $s$ divided by the mean $m$, assumed to be equal for the two data sets), the required discrimination $d$ (difference between the two means) and $t$ (crit, $n$ ), the critical value of $t$ (for $2 n-2$ degrees of freedom, which is 1.96 for $P=0.05$ in a two-sided test for large $n$ and e.g. 2.10 for $n=10$ ).

$$
d / m=\left[(s / m) 2^{1 / 2} t(\text { crit }, n)\right] / n^{1 / 2}
$$

The equation gives a bare minimum number of replicates, as it refers to a design of power 0.5 , i.e. in any experiment there is $50 \%$ chance that a significant result will be obtained if the actual difference between the two means is of size $d$. Fig. 3 shows the relationship.

Table 1 gives observed values of the coefficient of variation for grassland and cereals, as obtained in the past (mostly unpublished data of Dr J. J. Schuurman). The coefficient of variation normally is $30-50 \%$ in the upper layers (average $40 \%$ for $0-30 \mathrm{~cm}$ depth) and is slightly higher in deeper soil layers (average $48 \%$ for 30-60 $\mathrm{cm}$ depth). Considering the large difference between root weights in these layers, which often is more than an order of magnitude, the coefficient of variation is remarkably constant. In fields with marked heterogeneity in the composition of the soil profile, the variation in root samples is larger as well.

Fig. 3 shows that for apparently normal coefficients of variation of $40 \%$, we need at least 25 replicate samples per treatment if we want to distinguish $22 \%$ differences between two means, or 10 samples for $35 \%$ differences. 
Table 1. Coefficient of variation of root dry weight in auger samples of grassland and cereals (largely unpublished data obtained at the Institute for Soil Fertility). Coefficients are expressed as percentages; $n=$ number of samples, $\emptyset$ is diameter of auger $(\mathrm{cm})$.

\begin{tabular}{|c|c|c|c|c|c|c|c|c|c|c|c|c|c|c|c|c|c|c|c|c|}
\hline \multirow{3}{*}{\multicolumn{2}{|c|}{ Depth $(\mathrm{cm})$}} & \multicolumn{8}{|c|}{ Grassland } & \multicolumn{11}{|c|}{ Cereals } \\
\hline & & \multirow[t]{2}{*}{1} & \multirow[t]{2}{*}{2} & \multirow[t]{2}{*}{3} & \multirow[t]{2}{*}{4} & \multirow[t]{2}{*}{5} & \multirow[t]{2}{*}{6} & \multirow[t]{2}{*}{7} & \multirow[t]{2}{*}{8} & \multirow[t]{2}{*}{9} & \multirow[t]{2}{*}{10} & \multirow[t]{2}{*}{11} & \multirow[t]{2}{*}{12} & \multirow[t]{2}{*}{13} & \multicolumn{3}{|c|}{14} & \multicolumn{3}{|c|}{15} \\
\hline & & & & & & & & & & & & & & & i & $b$ & $t$ & $\mathrm{i}$ & $b$ & $t$ \\
\hline & $n \rightarrow$ & 100 & 100 & 20 & 20 & 20 & 20 & 20 & 50 & 50 & 25 & 25 & 20 & 20 & 4 & 4 & 8 & 4 & 4 & 8 \\
\hline & $\phi \rightarrow$ & 7 & 4 & 7 & 7 & 7 & 7 & 7 & 4 & 4 & 7 & 7 & 7 & 7 & 7 & 7 & 7 & 7 & 7 & 7 \\
\hline $0-5$ & & & & 34 & 30 & 51 & 45 & 41 & 29 & 43 & 30 & 43 & & & 41 & 89 & 100 & 47 & 32 & 103 \\
\hline $5-10$ & & 33 & 38 & 334 & 36 & 33 & 48 & & 29 & 37 & & & & & 83 & 56 & 73 & 105 & 36 & 70 \\
\hline $10-20$ & & & & 30 & 45 & 44 & 30 & 55 & 36 & 50 & 34 & 27 & 47 & 40 & 43 & 32 & 37 & 36 & 31 & 33 \\
\hline $20-30$ & & & & 36 & 41 & 40 & 38 & 75 & 43 & 56 & 31 & 28 & & & 73 & 64 & 65 & 42 & 44 & 43 \\
\hline $30-40$ & & & & 35 & 41 & 55 & 38 & 53 & 35 & 38 & 36 & 37 & 43 & 45 & & & 69 & & & 63 \\
\hline $40-50$ & & 35 & 41 & 152 & 31 & 48 & 47 & 59 & 46 & 31 & 35 & 29 & & & & & 78 & & & 59 \\
\hline $50-60$ & & & & 44 & 44 & 54 & 54 & 76 & 51 & 53 & 39 & 35 & 50 & 54 & & & 46 & & & 46 \\
\hline $60-70$ & & & & & & & & 100 & 56 & 39 & 56 & 47 & & & & & 48 & & & 45 \\
\hline $70-80$ & & & & & & & & 85 & 75 & 46 & 53 & 53 & & & & & 43 & & & 62 \\
\hline $80-90$ & & & & & & & & 76 & & & 61 & 63 & & & & & 72 & & & 106 \\
\hline $90-100$ & & & & & & & & 76 & & & 44 & 50 & & & & & 54 & & & 125 \\
\hline
\end{tabular}

Details: 1 and 2 homogeneous grassland (1949), 3 and 4 young grassland (at Gilze, 1966) at 150 resp. 300 $\mathrm{kg} \mathrm{N}^{-1}$ year $^{-1}, 5$ and 6 the same fields as 3 and 4 four years later (June, 1970), 7 established grassland (1976), 8 and 9 established grassland: root dry weight and root counts (estimates), respectively (Schuurman \& Knot, 1957), 10 and 11 oats: root dry weight and root counts (estimates), respectively (ibid.), 12 and 13 winter wheat on cracking clay soil (Biddinghuizen, May and June 1977), 14 and 15 spring wheat on clay loam and sandy loam, respectively (Ulrum, 1957): $i=$ sample in the row, $b=$ sample between rows, $t=$ equal number of $i$ and $b$ type sample combined.

\section{Cereals}

Table 1 shows that variation coefficients for root dry weight of cereals are slightly higher than those for grassland (average $45 \%$ for $0-30 \mathrm{~cm}$ depth and $51 \%$ for $30-60 \mathrm{~cm}$ depth). In the upper soil layers conventionally equal numbers of samples are taken 'in the row' and 'between rows'. When these two types of samples are handled as one set of data, the coefficient of variation rises sharply for the top $5 \mathrm{~cm}$, indicating that the two subsets are basically different.

The conventional scheme for sampling, distinguishing these two positions, is not satisfactory, however. There is a high concentration of roots immediately around the plant in the top soil (Fig. 4A). For a row distance of $25 \mathrm{~cm}$ the representative half of the unit soil strip is $12.5 \mathrm{~cm}$ wide. Fig. $4 \mathrm{D}$ shows that this strip can be covered in various ways by auger samples. To the conventional scheme $\mathrm{C}$ a third sampling position can be added, as shown in scheme $A$. The three sampling positions in this scheme A1, A2 and A3 carry a weight of 1:2:1. Scheme B covers the representative strip with two auger positions. A scheme $D$ can be introduced using the same sampling positions as scheme $C$, but giving a 1:3 weight to the two positions D1 and D2 (for justification see below). Shaded areas in Fig. 4D indicate the areas not covered by the samples; these areas are under-represented and cause the estimated average root density to be biased if they have a root density below or above the true aver- 
A

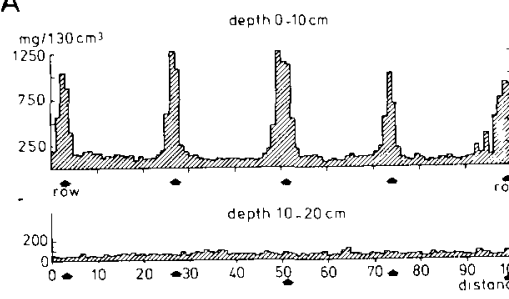

relative

C<smiles>O=c1cccco1</smiles>
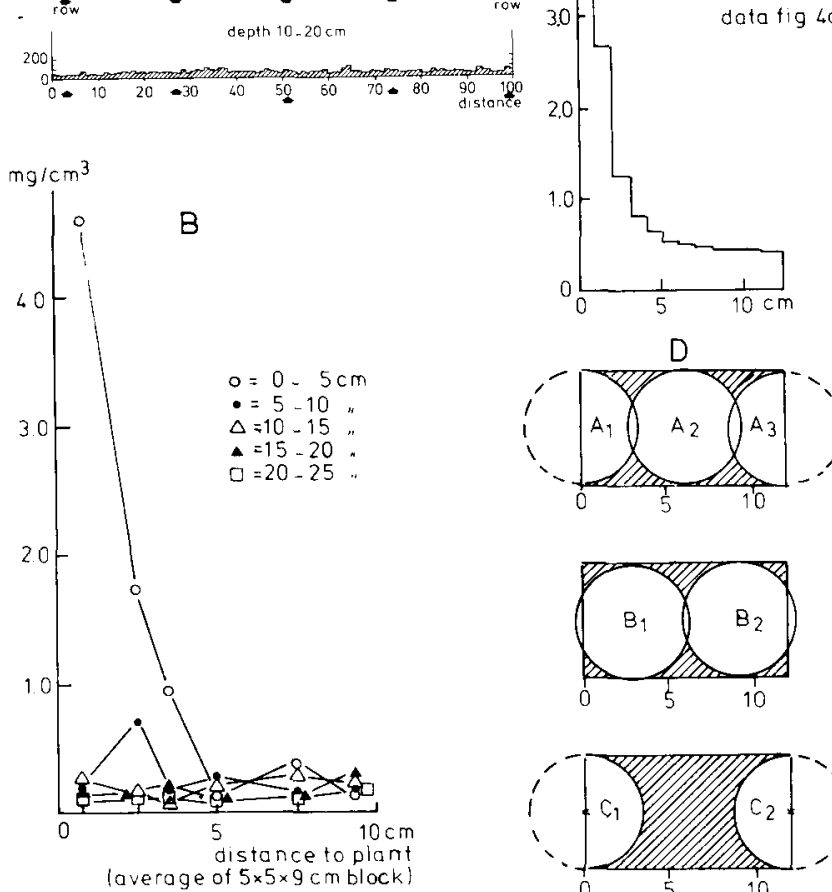

$\Delta=10-15$

$=15-20$
$=20-25$
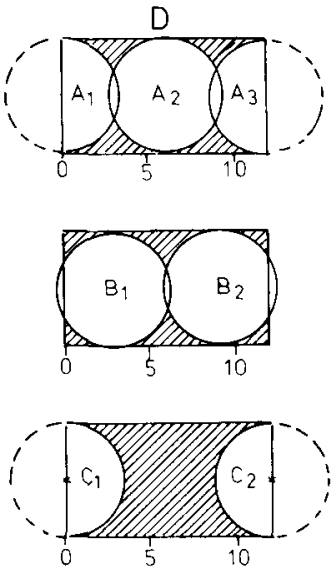

Fig. 4. Distribution of cereal roots around the plant and possible sampling schemes. A: detailed data of root weight in $1 \mathrm{~cm}$ blocks (Jonker, 1958), for winter wheat on a loam soil (Northeast Polder). B: unpublished data of Knot for rye on a sandy soil (spring 1957), root weights of $5 \mathrm{~cm} \times 5 \mathrm{~cm} \times 9 \mathrm{~cm}$ blocks plotted against their midpoint distance to the plant. C: summary of data in A for the top $10 \mathrm{~cm}$. D: possible ways to cover the representative strip of soil with auger samples ( $7 \mathrm{~cm}$ diameter), as seen from above. Shaded areas are not covered by the sampling scheme, dotted areas are overlap.

age. The magnitude of this bias can be calculated for given root distributions. The root distribution of Fig. $4 \mathrm{C}$ can be adequately described as a curve fitted by a negative exponential function of the distance to the plant

$$
R(x)=\mathrm{a}+\mathrm{be}^{-\mathrm{cx}}
$$

with the following values for the parameters $\mathrm{a}, \mathrm{b}$ and $\mathrm{c}: \mathrm{a}=0.4 \mathrm{mg} \mathrm{cm}^{-3} ; \mathrm{b}=5 \mathrm{mg}$ $\mathrm{cm}^{-3}$ and $\mathrm{c}=0.7 \mathrm{~cm}^{-1} . R(x)$ is the root density at distance $x$ from the plant $(\mathrm{mg}$ $\mathrm{cm}^{-3}$ ). Using this formula the various sampling schemes can be tested, by integrating (2) over the respective circles and dividing the result by the surface area of the 
circle. Average root densities per sample position obtained in this way can be averaged according to the sampling scheme. The result of this calculation, $\hat{R}$, can be compared to the true average density $\bar{R}$ to obtain the percentage bias according to

$$
\% \text { bias }=100(\hat{R}-\bar{R}) / \bar{R}
$$

The bias has been calculated for the various schemes for the root distribution shown in Fig. 4C, but also for other cases by changing the values of parameters a, b and c. Parameters $a$ and $b$ appear to be relatively unimportant, but the value of parameter $\mathrm{c}$ does affect the $\%$ bias. High values of $\mathrm{c}$ correspond to a very pronounced decrease of root density with increasing distance from the plant, low values to flat root distributions (and consequently little chance of biased results). Fig. 5A shows four types of root distribution, characterized by the parameter $\mathrm{c}$. Results for the \% bias for the four distributions of Fig. 5A are shown in Table 2.

Schemes A and B appear to be reasonably reliable methods, scheme D is acceptable for peaked root distributions, but not for relatively flat root distributions $(c=0.1$ or 0.4$)$. Scheme B is not very safe in its practical application as the exact position of the first sample does affect the result considerably and the degree of over-

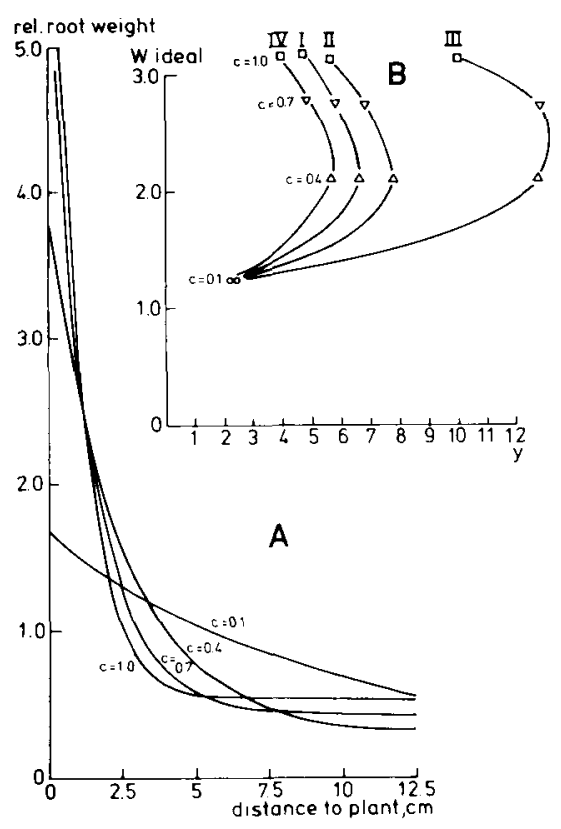

Fig. 5. A: relative root distribution for four values of parameter $c(a=0.45$ and $b=5.1)$; the line for $c=$ 0.7 has been curve-fitted to the data of Fig. 4c. B: ideal weighting factor $w$ for sample D2, for various root distributions, compared to the ratio $y$ of sample D1 to D2. The lines I-IV refer to root distributions with the following parameters: $\mathrm{I} a=0.45, \mathrm{~b}=5.1 ; \mathrm{II} \mathrm{a}=0.40, \mathrm{~b}=5.5 ; \mathrm{III} \mathrm{a}=0.20, \mathrm{~b}=5.5 ; \mathrm{IV} \mathrm{a}=0.50$, $\mathrm{b}=4.5$. 
Table 2. Percentage bias of the sampling schemes of Fig. 4D when tested on four types of root distribution (Equation 1); $c=0.7$ being a curve-fit to the data of Fig. $4 \mathrm{C}$.

\begin{tabular}{lllll} 
& $c=0.1$ & $c=0.4$ & $c=0.7$ & $c=1.0$ \\
Scheme A & +0.0 & +1.3 & +2.7 & +3.4 \\
Scheme B & -0.1 & -1.3 & -2.4 & -3.1 \\
Scheme C & +4.3 & +36.0 & +49.0 & +50.0 \\
Scheme D & -16.0 & -14.0 & -3.3 & +1.4 \\
\hline
\end{tabular}

lap with the row is not defined very sharply. The conventional scheme $\mathrm{C}$ can give large overestimates of around $50 \%$. Using the data for the two sampling positions and introducing a 1:3 weighting of the results (scheme $D$ ), the bias can be reduced considerably, although there may be a risk of underestimation of about $15 \%$. The magnitude of the bias with scheme $\mathrm{C}$ depends on the steepness and on the degree of non-linearity of the root distribution with distance. When the exact root distribution is known, an ideal weighting factor can be calculated which gives unbiased results for re-calculation of data obtained with inadequate sampling schemes. For the data of Fig. 4C this is 2.9. In practice, however, we do not know the complete root distribution, only the ratio $y$ of root weight in the row to root weight between rows, $y=R(\mathrm{C} 1) / R(\mathrm{C} 2)$. This ratio $y$ is not sufficient for determining an ideal $w$, as can be seen from Fig. 5B. For different root distributions, obtained by modifying parameters $\mathrm{a}, \mathrm{b}$ and $\mathrm{c}$ around the curve-fitted values, the relationship between $y$ and ideal $w$ is not constant (in fact, $w$ is dominated by parameter $\mathrm{c}$, and $y$ by parameters a and b). For correction of old data a value for $w$ in the range 2.5-3.0 seems to be the best choice, unless detailed information on root distribution is available.

The magnitude of the bias in root data obtained with the conventional scheme $\mathrm{C}$ can be estimated from the ratio $y$ :

$$
\% \text { bias }=100(w-1)(y-1) / 2(y+w)
$$

Table 3 gives some calculations of the $\%$ bias according to this formula for a range of $y$ values and for three assumed 'ideal $w$ ' values. The percentage bias exceeds $40 \%$ when $y$ is 5 or more. Table 4 gives a summary of available data on $y$ for row crops.

As the data in Table 4 indicate that a value of $y=5$ is a conservative estimate, the bias of the conventional sampling scheme for cereals can be taken to be around $50 \%$ for the important $0-10 \mathrm{~cm}$ layer. As this layer often contains $70 \%$ of the total root weight, errors made in estimating total root weight for expressing root:shoot ratios may be $35 \%$.

Below a depth of $10 \mathrm{~cm}$ the difference between $\mathrm{R}(\mathrm{C} 1)$ and $\mathrm{R}(\mathrm{C} 2)$ becomes small, though not always negligible (Table 4). Fig. 4B suggests that the difference between the two sampling positions can in fact be restricted to the $0-5 \mathrm{~cm}$ layer, the $5-10 \mathrm{~cm}$ layer having a peak density a few centimetres away from the plant. This peak can be considered a consequence of the preferential orientation of branch and 
Table 3. Percentage bias resulting from the use of the conventional sampling scheme $C$ for row crops, for various types of root distribution, characterized by the ideal weighting factor $w$ (which is not known in most cases) and $y$ (which is known, Table 4).

\begin{tabular}{rccc}
\hline$y$ & $w=2$ & $w=2.5$ & $w=3$ \\
1 & 0 & 0 & 0 \\
2 & 13 & 17 & 20 \\
5 & 29 & 40 & 50 \\
10 & 38 & 54 & 70 \\
20 & 43 & 63 & 83 \\
\hline
\end{tabular}

Table 4. Ratio of root dry weight 'in the row' and 'between rows' samples for various crops (largely unpublished data obtained at the Institute for Soil Fertility between 1949 and 1962). The distance between rows was $25 \mathrm{~cm} ; y$ is the mean of $n$ data sets, $r$ is the range.

\begin{tabular}{|c|c|c|c|c|}
\hline & \multicolumn{4}{|c|}{ Depth $(\mathrm{cm})$} \\
\hline & $0-10$ & $10-20$ & $20-30$ & $30-40$ \\
\hline \multirow[t]{2}{*}{ Winter wheat $n=4$} & $y 12.8$ & 1.33 & 1.10 & 1.09 \\
\hline & $r \quad 7.5-18$ & $0.79-1.9$ & $0.76 \cdot 1.3$ & $0.80-1.6$ \\
\hline \multirow[t]{2}{*}{ Spring wheat $n=11$} & $y \quad 9.77$ & 1.08 & 1.08 & 1.12 \\
\hline & $r \quad 2.8-26$ & $0.82-1.3$ & $0.74-1.4$ & $0.8-1.4$ \\
\hline \multirow[t]{2}{*}{ Oats $n=5$} & $\begin{array}{ll}y & 6.56\end{array}$ & 1.20 & 1.2 & 1.1 \\
\hline & $r 2.4-13$ & $0.84-2.0$ & $1.0-1.4$ & $0.9-1.4$ \\
\hline Rye $n=1$ & 6.9 & 0.88 & - & - \\
\hline \multicolumn{5}{|l|}{ Green manures } \\
\hline Various Leguminosae & $r \quad 1.7-6.0$ & $0.98-3.1$ & $1.1-1.9$ & - \\
\hline Various Cruciferae & $r \quad 1.8-20$ & $0.69-1.9$ & $0.58-1.1$ & - \\
\hline Various grasses & $r \quad 1.6-5.2$ & $0.71-1.1$ & $0.82-0.91$ & - \\
\hline
\end{tabular}

nodal roots (compare Fig. 2), causing a 'gap' directly under the plant. At lower depth no systematic differences in root density are evident.

If we take root length rather than root weight as the main parameter of root density, the differences between sampling positions are much less pronounced, as shown for example in Table 5 for a winter wheat crop. Just around the plant, where root weight is very high, the ratio between root length and root weight is very low. This ratio is apparently similar at a vertical and a horizontal distance of $10 \mathrm{~cm}$ from the plant base. The ratio of root length and root dry weight depends on root diameter and dry matter content, both of which may be expected to be high just around the plant. For all positions the coefficient of variation is much smaller for root length per unit dry weight than for root dry weight per unit volume of soil (about $15 \%$ and about $30 \%$, respectively). This difference is not surprising as the first applies to a 'quality' of the roots and the second to a 'quantity'.

\section{Sugar-beet}

Fig. 6 gives three possible schemes for subsampling the representative quart of the 'unit soil area' of plants with a spacing as is common for sugar-beet $(30 \mathrm{~cm} \times 50 \mathrm{~cm}$, 
Table 5. Results of sampling in winter wheat $(5$ samples per position, $\mathrm{a}=$ average, $\mathrm{cv}=$ coefficient of variation (\%), columns a, $\mathrm{c}$ and e refer to 'in the row' samples, $\mathrm{b}, \mathrm{d}$ and $\mathrm{f}$ to 'between rows' samples.

\begin{tabular}{|c|c|c|c|c|c|c|c|c|c|}
\hline \multirow[t]{2}{*}{$\begin{array}{l}\text { Depth } \\
(\mathrm{cm})\end{array}$} & \multicolumn{3}{|c|}{$\begin{array}{l}\text { Dry weight } / \mathrm{cm}^{3} \text { soil } \\
\left(\mathrm{mg} \mathrm{cm}^{-3}\right)\end{array}$} & \multicolumn{3}{|c|}{$\begin{array}{l}\text { Length } / \mathrm{cm}^{3} \text { soil } \\
\left(\mathrm{cm} \mathrm{cm}^{-3}\right)\end{array}$} & \multicolumn{3}{|c|}{$\begin{array}{l}\text { Length/dry weight } \\
\text { (cm mg-1) }\end{array}$} \\
\hline & a & b & $a / b$ & c & d & $\mathrm{c} / \mathrm{d}$ & $\mathrm{e}$ & f & $e / f$ \\
\hline \multirow{2}{*}{$\begin{array}{ll}0-5 & \mathrm{a} \\
& \mathrm{cv}\end{array}$} & 1.82 & 0.083 & 21.9 & 4.61 & 2.02 & 2.3 & 2.65 & 24.5 & 0.11 \\
\hline & 27 & 12 & & 13 & 9 & & 24 & 5 & \\
\hline \multirow{2}{*}{$\begin{array}{r}5-10 a \\
c v\end{array}$} & 0.27 & 0.117 & 2.3 & 3.79 & 3.30 & 1.15 & 14.0 & 27.5 & 0.51 \\
\hline & 32 & 32 & & 33 & 40 & & 13 & 17 & \\
\hline \multirow{2}{*}{$\begin{array}{r}10-20 \mathrm{a} \\
\mathrm{cv}\end{array}$} & 0.091 & 0.105 & 0.87 & 2.32 & 2.70 & 0.86 & 25.2 & 25.8 & 0.98 \\
\hline & 40 & 24 & & 44 & 26 & & 7 & 13 & \\
\hline
\end{tabular}

$7 \mathrm{~cm}$ diameter auger samples). A fourth method $\mathrm{D}$ can be constructed by using the sample results of method $\mathrm{C}$ but giving double weight to positions $\mathrm{C} 3$ and $\mathrm{C} 4$ when calculating average root density. A method $\mathrm{E}$ can be introduced, comparable to method B but without sampling positions B3 and B4.

To test the accuracy of these schemes in determining average root density, they were tested in four theoretical root distributions I-IV, as defined in Fig. 6. These theoretical root distributions are assumed to be radially symmetrical around the plant with unimpeded overlap between roots of neighbouring plants. The first type of root distribution, which may be expected in young plants, is the most heterogeneous and therefore gives the most rigorous test of the sampling schemes. The other root distributions, with more overlap, are more homogeneous and easier to cover. Results for the three sampling methods are shown in Table 6.
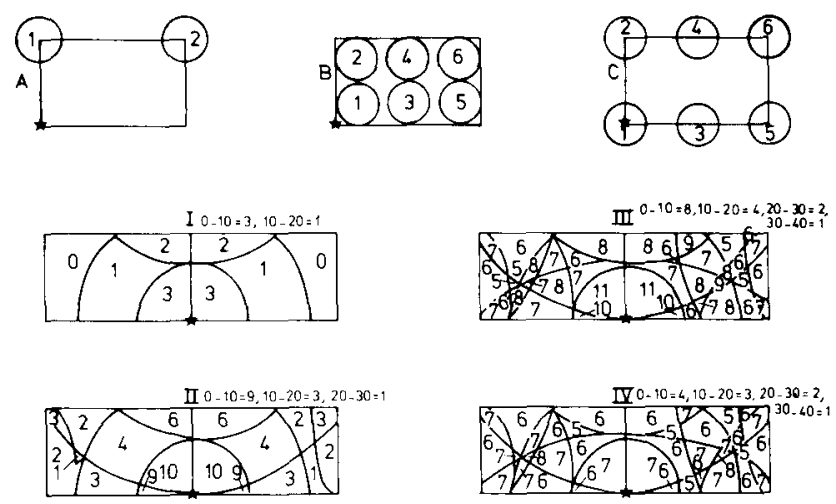

Fig. 6. Three sampling schemes (A, B and C) for sampling the representative quarter of the unit soil area for a $30 \mathrm{~cm} \times 50 \mathrm{~cm}$ plant spacing (plant denoted by asterisk). Four hypothetical root distributions (I to IV) with which the three sampling schemes have been tested are shown in top view; the root densities per ring around the plant are listed above each figure, root densities of neighbouring plants have been added for each position. 
Table 6. Results of sampling schemes shown in Fig. 6, applied to the four types of root distribution in the same figure. Estimated average root density is given as percentage difference from the true average.

\begin{tabular}{lllrll}
\hline Root distribution & \multicolumn{5}{l}{ Sampling scheme } \\
\cline { 2 - 6 } & A & B & C & D & E \\
I & -26 & -8 & -9 & -10 & -7 \\
II & -8 & -3 & 0 & -7 & +8 \\
III & -8 & -1 & -1 & -2 & +2 \\
IV & -3 & -1 & -1 & -2 & +1 \\
\hline
\end{tabular}

Method A obviously is the least reliable one (it gives a $26 \%$ underestimate for root distribution I). Scheme $\mathrm{B}$ and $\mathrm{C}$ give a 8 or $9 \%$ underestimate for root distribution I but are reliable otherwise. Scheme $\mathrm{D}$ is performing worse than scheme $\mathrm{C}$ for root distribution II, a general justification of this conclusion being impossible. Scheme E gives a $7 \%$ underestimate for root distribution I and $8 \%$ overestimate for root distribution II; the use of this scheme may therefore give fluctuations in the results which are unrealistic. For practical reasons scheme $C$ cannot be used in sugar-beet, as it would comprise the tap root, so scheme B is the best choice.
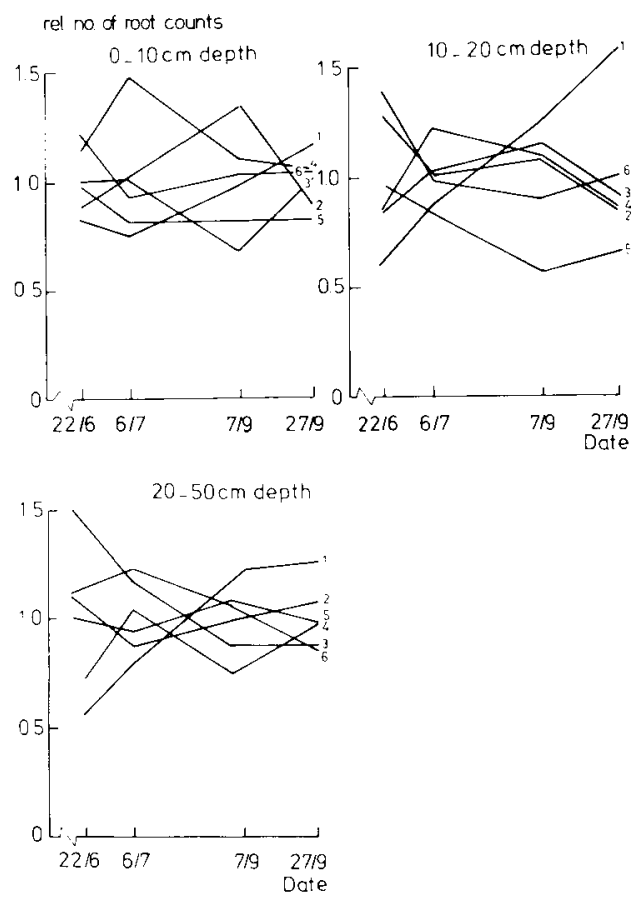

Fig. 7. Relative distribution of sugar beet root counts over six sampling positions (Fig. 6B); every point is the mean of 8 samples. 
Method B was used in 1982 to quantify root systems of sugar-beet plants by the core-breaking method (as used by Drew \& Saker, 1980). Results for the various positions are given in Fig. 7. Differences between sampling positions may be considerable, although the pattern is not always clear. Surprisingly, root counts at position B1, just around the plant, are relatively low initially, rising steadily at all depths. This might be due to the method used, which counts vertical roots preferentially and may underestimate the largely horizontal branch roots from the main axis at this position (later the second-order branch roots will have a random orientation). The differences between sampling positions do indicate that sampling schemes not covering the unit soil area may miss important information.

Neglecting sample position, the coefficients of variation are high compared with values in Table 1: about $65 \%$ for the plough-layer and more than $100 \%$ below a depth of $30 \mathrm{~cm}$. This difference may be due either to a difference in method or to a difference in crop. In grassland the coefficient of variation of root estimates in the core-break method was found to be slightly higher than that for root weights in auger samples, the difference being small (Table 1). The high values for the sugar-beet coefficients of variation therefore probably reflect a highly heterogeneous root distribution, rather than an inaccurate method. The high heterogeneity of root distribution in sugar-beet is probably related to the beard-like branching pattern from the main axis. As the direction of the beard can only be predicted by excavation, a large number of samples and an adequate sampling scheme constitute the only way to overcome these problems.

\section{Potato}

For potato, a sampling scheme similar to scheme $C$ in Fig. 6 appears to be most practical, accommodating for ridge height as shown in Fig. 8. Scheme B may be theoretically more adequate, but its exact sampling positions are harder to define in the field. Positions of scheme $\mathrm{C}$ may be termed: on top of the ridge, halfway down the slope and down in the trough, both inbetween two plants and below a plant.

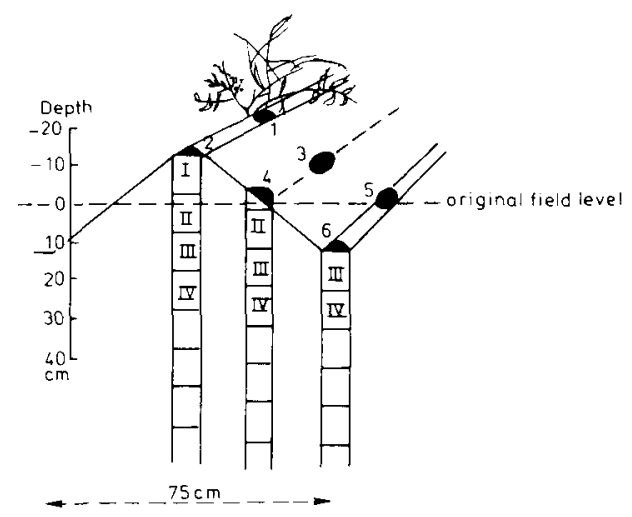

Fig. 8. Sampling scheme for a potato ridge, comparable to scheme $\mathrm{C}$ in Fig. 6. 


\section{Sampling with pinboard techniques}

\section{Theory}

Pinboard samples generally give a better overall view of the distribution of roots in the soil than auger samples, although the method is less reliable quantitatively as roots may change position to some extent during washing and subsequent handling of samples, or may be lost due to breaking off. Pinboard samples can be used to describe distribution of roots in terms of branching pattern. When we try to estimate the total size of the root system of a plant from the slice of soil in our sample some difficulties arise.

For grassland where the main orientation of roots is vertical with only small branch roots in a horizontal direction, problems are small. Root densities on the pinboard are reasonably safe estimates of root density per unit volume of soil, except for root sections lost during washing and incomplete removal of all dead organic matter from the top layers. For cereals pinboard samples taken perpendicular to the row direction give information on root densities per unit volume of soil in all relevant positions. In sugar-beet the heterogeneity of root branching from the main axis (the 'beard') causes problems, as pinboards either contain a very high or a very low number of primary branch roots. A high number of replicates is required to overcome these problems. With potatoes, problems arise from the fact that the slice of soil in the pinboard sample, usually including a plant, is only part of the unit soil area and nor representative as such, whether the samples are taken 'in the row' or perpendicular to it.

A rough estimation procedure is possible when circular symmetry of the root system around the plant can be assumed, as shown in Fig. 9 for potato. By subsampling, after washing and removal of roots of neighbouring plants, we can use pinboard samples to estimate root density as a function of distance to the plant (horizontal and vertical). These density estimates can be multiplied by the volume of soil in the corresponding ring of soil around the plant and added to give total root weight per plant (or root length or surface area if this is our parameter of root density). The total size of the root system per plant can be calculated as:

$$
\sum_{j=1}^{h} \sum_{i=1}^{n} R_{i j} \pi\left(i^{2}-(i-1)^{2}\right) \cdot 100=100 \pi \sum_{j=1}^{h} \sum_{i=1}^{n} R_{i j}(2 i-1)
$$

$R_{\mathrm{ij}}$ is root density at horizontal distance $i$ from the plant at depth $j, h$ is root depth and $n$ is horizontal dispersion (all expressed per $10 \mathrm{~cm}$ ).

Although conceptually simple, this method theoretically requires the use of sector-shaped samples. Instead of the normal pinboards with pins of constant length, boards might be constructed with very short pins on one side (near the plant) and long pins on the other side. When we use conventional pinboards we have to face the bias resulting from the use of rectangular samples to estimate root density in circles. Fig. 10 illustrates the problem. The rectangle ABCD is not a good estimator of the density in the inner circle, as area V and VI do in fact belong to the second ring, 


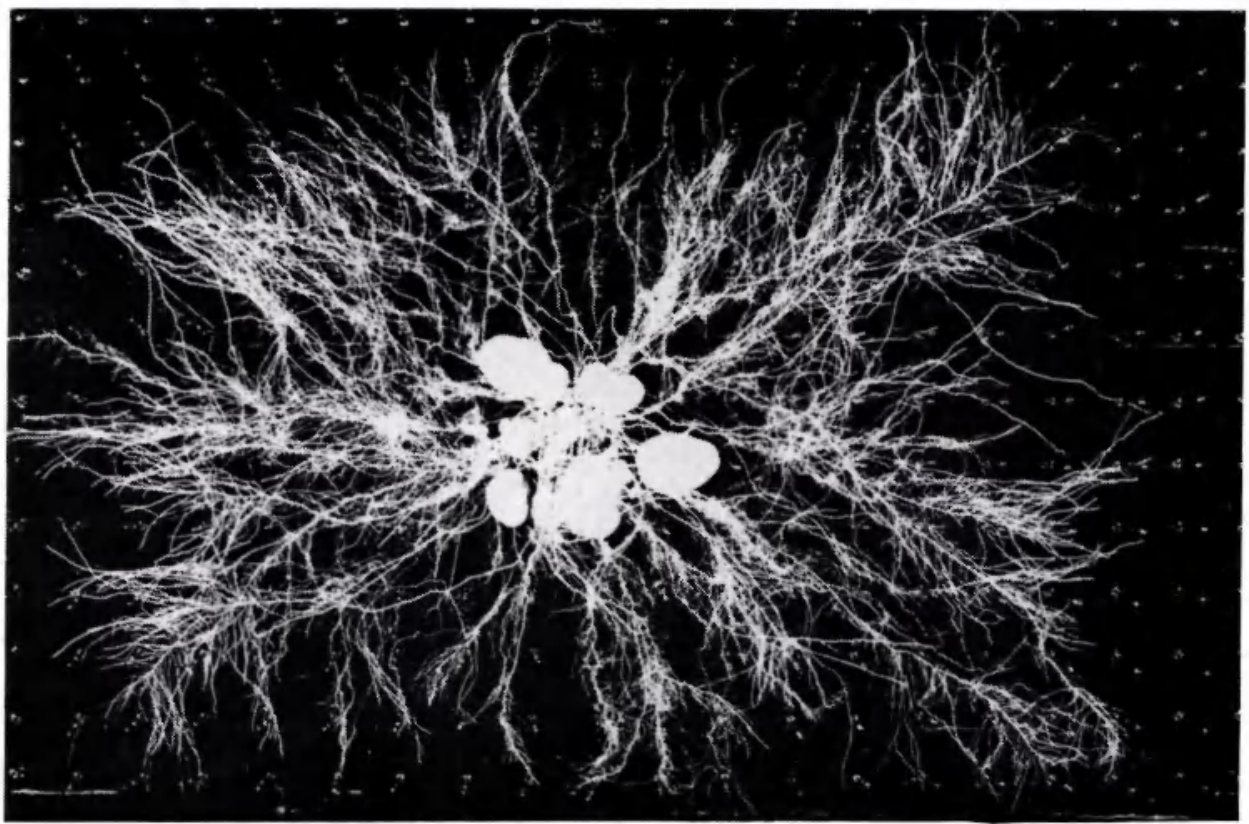

Fig. 9. Top view of a potato root system, washed out on a pinboard, showing the radial symmetry or spider shape.

while area IV is missing. In the second ring the rectangle CDEF is not a good estimator, because area VI is missing, while area IX and $X$ are superfluous and area VII is overrepresented. If root density is a linear function of distance to the plant (at least for local approximation) we can compare the average distance to the plant for the blocks with that for sector segments to get some idea of the possible bias. Table 7 shows the results of such a comparison, where the average distance for the sector segments has been derived mathematically and the average distance for the blocks has been measured with a fine grid for subsampling. When pinboards are used with pins of $15 \mathrm{~cm}$, as in Fig. 10, the average distance for the blocks is surprisingly similar to that for sector segments, so bias will be negligible. For $10-\mathrm{cm}$ pins the average distance is lower in the blocks, so root densities will probably be overestimated, the severity of this bias being dependent on the unknown relationship between root density and distance to the plant.

The discussion given here in terms of absolute distances can be based on relative distances as well, with the ratio between pin length and the radius of the circles as the main parameter. Pinboards with pins of $10 \mathrm{~cm}$ can be used without much bias if the calculations are based on subsamples and circles of 6.7 instead of $10 \mathrm{~cm}$ (this corresponds to $15-\mathrm{cm}$ pins and $10-\mathrm{cm}$ circles).

Ridge structure in potatoes complicates the method, as not all rings are complete. Samples in the row and perpendicular to the row can be used to compare root densities as a function of distance to the plant in the two directions. If the difference 
Table 7. Average distance $(\mathrm{cm})$ to the plant for sector segments and rectangular samples (see Fig. 10).

\begin{tabular}{llll}
\hline & Ring 1 & Ring 2 & Ring 3 \\
& 6.67 & 15.56 & 25.33 \\
$\begin{array}{l}\text { Sector segment } \\
\text { Rectangular sample }\end{array} \quad$ & & & \\
$\quad 10 \mathrm{~cm} \times 10 \mathrm{~cm} \times 15 \mathrm{~cm}$ & 6.70 & 15.53 & 25.26 \\
$\quad \begin{array}{l}\text { Rectangular sample } \\
\quad 10 \mathrm{~cm} \times 10 \mathrm{~cm} \times 10 \mathrm{~cm}\end{array}$ & 5.84 & 15.20 & 25.08 \\
\hline
\end{tabular}

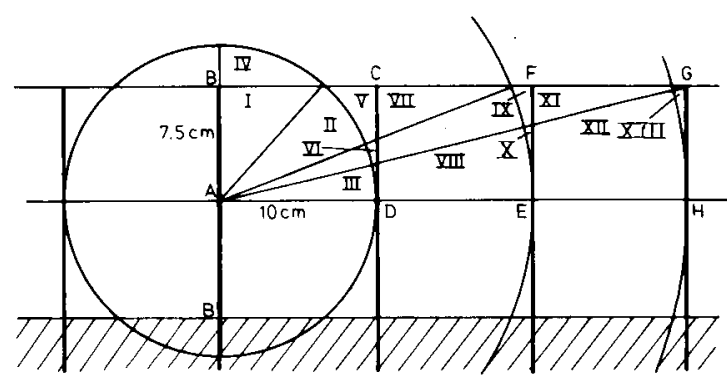

Fig. 10. Top view of a pinboard sample (with pins of $15 \mathrm{~cm}$ length), with the plant at $A$. The sample can be cut into blocks of $10 \mathrm{~cm} \times 10 \mathrm{~cm} \times 15 \mathrm{~cm}(10 \mathrm{~cm}$ depth) which consist of two equal halves. Root density in half-block $A B C D$ is not an unbiased estimate of the average density in the first ring around the plant; see text for further explanation.

between the two is small enough to be neglected, the calculation of Equation 5 can be performed after correction for the part of each ring which is actually present in the ridge. For example, for the geometry of Fig. 8 correction factors are: 1.0, 0.74, $0.43,0.29$ and 0.21 for ring 1 to 5 , respectively, for $-10 \mathrm{~cm}$ to $0 \mathrm{~cm}$ depth, and 1.0 , $1.0,0.8,0.64$ and 0.42 , respectively, for 0 to $10 \mathrm{~cm}$ depth.

\section{Example}

As an example, data will be discussed for a potato experiment in 1979 in which split application of $\mathrm{N}$ fertilizer was tested in combination with sprinkler irrigation on a loam soil (Creil, Northeast Polder; for details see Schepers et al., 1984). At two dates pinboard samples were taken 'in the row'. After washing, roots of neighbouring plants were removed and the root system was cut to determine root densities per $10 \mathrm{~cm} \times 10 \mathrm{~cm} \times 15 \mathrm{~cm}$ subsample. Fig. 11 shows results for root dry weight. On the first date, late June, little difference in root development was found between the two $\mathrm{N}$ fertilizer levels (150 and $225 \mathrm{~kg} \mathrm{~N} \mathrm{ha}^{-1}$ ). One month later the plants of the split-application treatment $\left(150+75 \mathrm{~kg} \mathrm{~N} \mathrm{ha}^{-1}\right)$ had a root distribution different from that of the plants receiving $225 \mathrm{~kg} \mathrm{~N}$ as a single dose. Horizontal dispersion in the surface layers was increased in the latter case, especially in the irrigation treatment. This difference in root distribution causes a large difference in the outcome of Equation 5, when compared to the root mass on the pinboard (this ratio varies from 1.7 to 2.9 ). Results of calculations are presented in Table 8 . 


\section{SAMPLING SCHEMES FOR ESTIMATING ROOT DENSITY DISTRIBUTION}

Table 8. Results of calculation according to Equation 5 on data of Fig. 11.

\begin{tabular}{|c|c|c|c|c|c|c|c|c|}
\hline \multirow{2}{*}{$\begin{array}{l}\text { Depth } \\
\text { (cm) }\end{array}$} & \multirow{2}{*}{$\begin{array}{l}\text { date } \\
\text { treatment }\end{array}$} & \multirow{2}{*}{$\begin{array}{l}\rightarrow \\
\rightarrow\end{array}$} & \multicolumn{2}{|l|}{28 June } & \multicolumn{4}{|l|}{30 July } \\
\hline & & & $150+75$ & 225 & $150+75$ & $\begin{array}{l}150+75 \\
\text { irrig. }\end{array}$ & 225 & $\begin{array}{l}225 \mathrm{~kg} \mathrm{~N} \mathrm{ha}^{-1} \\
\text { irrig. }\end{array}$ \\
\hline \multicolumn{9}{|c|}{ Observed mg sample -1 $^{-1}$} \\
\hline$-10-0$ & & & 694 & 684 & 716 & 828 & 977 & 753 \\
\hline $0-10$ & & & 448 & 486 & 624 & 772 & 898 & 1199 \\
\hline $10-20$ & & & 72 & 97 & 223 & 375 & 289 & 487 \\
\hline $20-30$ & & & 0 & 0 & 73 & 193 & 73 & 107 \\
\hline $30-40$ & & & 0 & 0 & 11 & 15 & 0 & 0 \\
\hline total & & & 1214 & 1267 & 1674 & 2187 & 2237 & 2546 \\
\hline \multicolumn{9}{|c|}{ Estimated $m g$ plant ${ }^{I}$} \\
\hline$-10-0$ & & & 947 & 1029 & 1027 & 1345 & 1756 & 1313 \\
\hline $0-10$ & & & 868 & 875 & 1393 & 1558 & 2440 & 3522 \\
\hline $10-20$ & & & 235 & 206 & 675 & 1003 & 1088 & 2195 \\
\hline $20-30$ & & & 0 & 0 & 235 & 569 & 204 & 342 \\
\hline $30-40$ & & & 0 & 0 & 30 & 48 & 0 & 0 \\
\hline total & & & 2050 & 2110 & 3361 & 4523 & 5488 & 7372 \\
\hline Shoot:rc & tratio & & 9.1 & 9.6 & 15.9 & 14.1 & 11.2 & 7.3 \\
\hline
\end{tabular}

Root weight density

$\mathrm{mg} / 1.5 \mathrm{dm}^{3}$
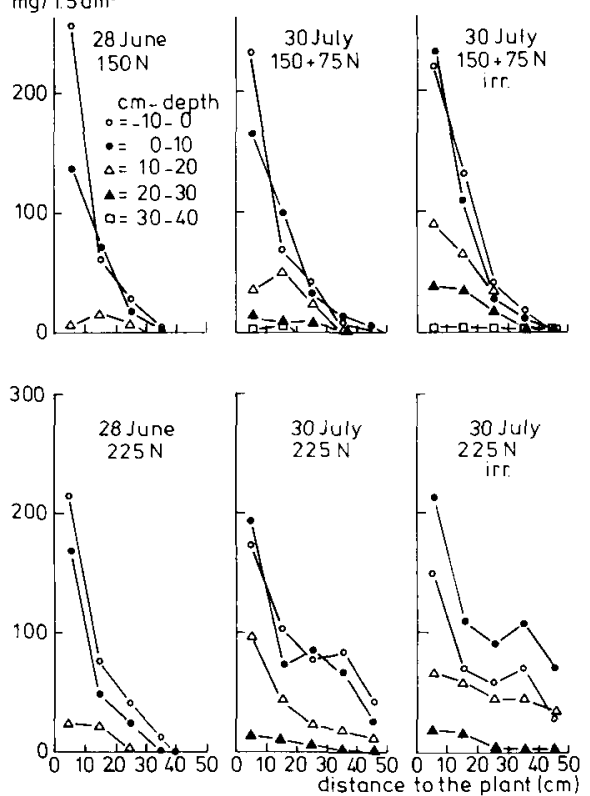

Fig. 11. Root weight density as a function of distance to the plant at various depths for a potato experiment with split $\mathrm{N}$ application and sprinkler irrigation at Creil, 1979.

Neth. J. agric. Sci. 33 (1985) 


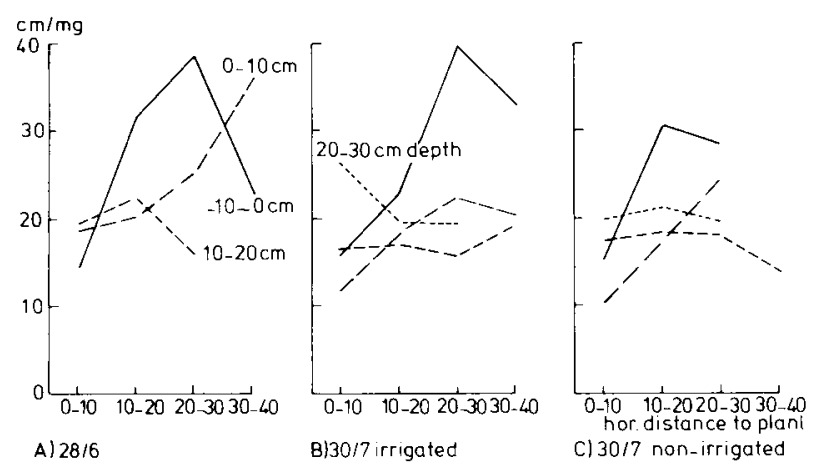

Fig. 12. Specific root length of potato roots according to their position in the soil for the samples of Fig. 11 ; each point is the mean of $2-4$ measurements.

The procedure used is quite sensitive to errors due to displacement of roots during and after washing on the pinboard, especially when roots of neighbouring plants are removed. For pinboards of $100 \mathrm{~cm}$ width and a plant distance in the row of $40 \mathrm{~cm}$, one pinboard sample will contain a slice of one plant's roots, two halves and twice the column at $0-10 \mathrm{~cm}$ horizontal distance to the plant. Total root weight of such a sample, where all roots are left undisturbed, can be used to check the root weight distribution measured for the central plant individually on replicate samples. Such a comparison for the data of Fig. 11 showed fair agreement, except for the irrigation treatment (especially at $225 \mathrm{~kg} \mathrm{~N} \mathrm{ha}^{-1}$ ), where root density in the column at $0-10 \mathrm{~cm}$ from the plant was apparently underestimated. This probably means that root density in the other zones was overestimated and thus total root weight was overestimated. The necessary check on symmetry in root distribution has not been made in this case.

As Fig. 12 shows, the ratio of root length/dry weight depends on the position in the soil. Generally this ratio increases with horizontal distance to the plant. High ratios indicate fine branching (or low dry-matter content of the roots). When Fig. 12B and $\mathrm{C}$ are compared an effect of sprinkler irrigation is apparent; it increased the ratio in the topsoil (or prevented the decrease with time found in drier soil). Auger samples taken in the field would miss most of this information because of the considerable overlap of root systems of neighbouring plants causing each sample to contain a mixture of roots.

\section{Profile wall observations}

Comparable to the extrapolation method for pinboard data, the assumption of radial symmetry can also be used for calculating root length per plant from counts of intersections with a profile wall. In field situations it may generally not be possible to identify roots belonging to a specific plant on intersections (unless a dye is introduced into the root system via the shoot), but in early stages of growth or for isola- 


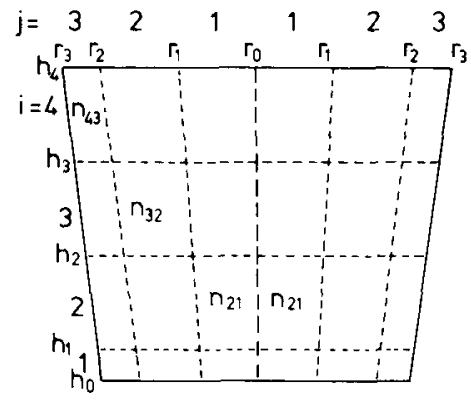

Fig. 13. Section through the centre of a round plant pot, showing areas in which roots can be counted for extrapolation to the total root length of the plant. Pot height $h$ and pot radius $r$ are divided into $p$ and $q$ parts respectively ( 4 and 3 in the example given).

ted trees overlap between root systems of neighbouring plants can be negligible. For pot plants the method can be directly applicable to observations on a section through the centre of the pot (see Fig. 13). By counting the number of roots seen per unit surface area in a sampling grid, root length per unit volume of soil can be estimated to be twice this value, under the assumption of root anisotropy (Melhuish \& Lang, 1971). Root density in each zone can be multiplied by the relevant soil volume, as before:

$$
L=2 \pi \sum_{i=1}^{p} \sum_{j=1}^{q} n_{i, j}\left(r_{i, j}^{2}-r_{i, j-1}^{2}\right)\left(h_{i}-h_{i-1}\right)
$$

with $n(i, j)$ as the number of roots observed per $\mathrm{cm}^{2}$ in the subsample at depth $i$ and radius $j$ and $r(0)=h(0)=0$.

Fig. 13 shows an application of the method in a pot experiment, which is slightly more complicated than the field situation, as the sample unit is not a rectangle. By taking $r(i, j)$ as the radius at $[h(i-1)+h(i)] / 2$, Equation 6 can be used as approximation. The subsamples should be small to reduce bias, especially at the bottom and sides of the pot where concentrations of roots may be expected to occur.

\section{Discussion}

It may be surprising that more than fifty years after the start of quantitative root research, elementary problems as the design of adequate sampling schemes have not been dealt with. In his survey of methods for studying root systems, Böhm (1979) discusses the number of samples required, but not their position. Jonker (1958) compared the results of auger sampling to monoliths (small blocks in the unit soil area) and concluded that 'root density estimates obtained by auger methods do not give adequate representation of root distribution in the field'. Jonker could not explain the discrepancies found as we can now, considering the inadequate sampling schemes used for auger sampling and the variable losses of root dry weight in the 
various washing procedures (van Noordwijk \& Floris, 1979, Floris \& de Jager, 1981).

Published data on root weights in the field should be regarded with suspicion, because of the possibility of inadequate sampling schemes. This may be most relevant for the estimated input of organic matter into the soil in crop rotations. In some cases overestimates due to inadequate sampling schemes may compensate for the losses of root dry weight during washing and handling as both may be about $30 \%$.

Root length may be more homogeneously distributed in the soil than root weight (Table 5). Apart from obvious significance of this fact for the plant, it is important in root research methods, as it simplifies sampling problems. Root length determinations also suffer less from the losses of dry weight reported before, although loss of fine rootlets becomes more critical if we are interested in root length rather than root weight.

The coefficient of variation for root samples in the standard auger technique is at least $30 \%$. This means a high number of replicates is required if effects of experimental factors are to be measured with some accuracy. Root research with a lower number of replicates can be considered to be a waste of time and money, because no valid conclusions will be possible. Root counts on soil cores after breaking may have a higher coefficient of variation, perhaps twice as high, and still be a more efficient method in terms of time involved. If the coefficient of variation is $60 \%$ instead of $30 \%$, the number of replicates required to distinguish a $30 \%$ difference between two means is 33 instead of 10 (for a $20 \%$ difference 90 instead of 25). Because the time involved per sample differs considerably (about 120 samples per person per day for root counting or 3 for washing and cleaning), the root counting method is a better choice for many situations. Drew \& Saker (1980) performed calibration measurements for the core-break method using washed samples as a standard. They found calibration to be necessary at several growth stages of a cereal crop, probably because of changes in the orientation of roots, affecting the relative success of the core-break method. Information on variation per sample has not been given, so a comparison of the number samples required and time involved for the two methods is not possible.

A discussion of the number of replicates required for pinboard sampling, comparable to the discussion for auger samples, is not simple, as straightforward estimates of the coefficient of variation on this scale are rare (the usual procedure of sampling in duplicate from a single profile pit does not yield a reliable estimate of the variation). Variation on the scale of a pinboard sample cannot be directly related to that of auger samples by comparing respective sample sizes, as 'compensatory root growth' tends to reduce variation in size of the root system of a whole plant compared to that of a summation of small scale variation. On the other hand, pinboard samples relate to a single plant, so they depend on plant to plant differences in performance. The standard procedure of taking samples of 'representative' plants, as judged from above-ground performance, probably reduces sample variation, but carries a risk of introducing bias when real averages for the field are desired. The procedure of duplicate sampling probably represents a bare minimum for obtaining meaningful results in the field. 
Adequate choice of sampling schemes for all root-related events and organisms in the soil is obviously important. The schemes given here may help in such situations.

\section{Acknowledgements}

Ir G. Derksen (IWIS-TNO) gave critical comments and calculated the data for Table 2. Gerard Brouwer and Klaas Harmanny performed some of the root measurements reported here. Thanks are due to Dr B. W. Veen for critically reading the manuscript.

\section{References}

Böhm, W., 1979. Methods of studying root systems, Springer-Verlag, Berlin, 188 pp.

Brouwer, R., 1984. Functional equilibrium: sense or nonsense? Netherlands Journal of Agricultural Science 31: 335-348.

Drew, M. C. \& L. R. Saker, 1980. Assessment of a rapid method using soil cores, for estimating the amount and distribution of crop roots in the field. Plant and Soil 55: 297-305.

Floris, J. \& A. de Jager, 1981. Een schatting van het verlies aan drogestof en van de verandering in diameter van wortels van Engels raaigras (Lolium perenne) door bemonsteren, bewaren en spoelen. Instituut voor Bodemvruchtbaarheid, Rapport 1-81, 18 pp.

Jonker, J. J., 1958. Bewortelingsonderzoek en ondergrondbewerking in de Noordoostpolder. Dissertation, Agricultural University, Wageningen, $164 \mathrm{pp}$.

Melhuish, F. M. \& A. R. G. Lang, 1971. Quantitative studies of roots in soil. II. Analysis of nonrandom populations. Soil Science 112: 161-166.

Noordwijk, M. van, 1983. Functional interpretation of root densities in the field for nutrient and water uptake. In: Wurzelökologie und ihre Nutzanwendung (International Symposium Gumpenstein, 1982). Bundesanstalt Gumpenstein, Irdning, pp. 207-226.

Noordwijk, M. van \& J. Floris, 1979. Loss of dry weight during washing and storage of root samples. Plant and Soil 53: 239-243.

Schepers, J. H., G. J. G. Rauw \& J. Floris, 1984. Invloed beregening en N-bemesting op aardappelen, 1979 (IB 2503 (W.G. 27)); Wortelproduktie bij aardappelen (IB 2503 (W.G. 27)). Stichting Landbouwkundig Bureau Nederlandse Meststoffenindustrie, Verslag B 357, 12 pp.

Schuurman, J. J. \& M. A. J. Goedewaagen, 1971. Methods for the examination of root systems and roots. Pudoc, Wageningen, $186 \mathrm{pp}$.

Schuurman, J. J. \& L. Knot, 1957. Het schatten van hoeveelheden wortels in voor wortelonderzoek genomen monsters. Verslagen Landbouwkundige Onderzoekingen $63.14,31 \mathrm{pp}$.

Sokal, R. R. \& F. J. Rohlf, 1981. Biometry, 2nd ed. Freeman, San Francisco.

\section{Note added in proof}

To Eq. can be added that a more complete coverage of relevant theory and an algorithm for calculating $n$ for designs of higher power are given by Sokal \& Rohlf (1981, p. 263). 